\title{
Anomaly inflow on QCD axial domain-walls and vortices
}

\author{
Kenji Fukushima* and Shota Imaki ${ }^{\dagger}$ \\ Department of Physics, The University of Tokyo, 7-3-1 Hongo, Bunkyo-ku, Tokyo 113-0033, Japan
}

(Received 1 March 2018; published 6 June 2018)

\begin{abstract}
We study the chiral effective theory in the presence of quantum chromodynamics (QCD) vortices. Gauge invariance requires novel terms from vortex singularities in the gauged Wess-Zumino-Witten action, which incorporate anomaly-induced currents along the vortices. We examine these terms for systems with QCD axial domain-walls bounded by vortices (vortons) under magnetic fields. We discuss how the baryon and electric charge conservations are satisfied in these systems through interplay between domain-walls and vortices, manifesting Callan-Harvey's mechanism of anomaly inflow.
\end{abstract}

DOI: 10.1103/PhysRevD.97.114003

\section{INTRODUCTION}

Among various states realized on the phase diagram of matter out of quarks and gluons, which are fundamental particles in quantum chromodynamics (QCD), the region at high baryon density has been only poorly understood, and there are still diverging theoretical candidates for the true ground state (see Refs. [1-5] for comprehensive reviews of possible phases). If the baryon density is asymptotically high, we could perform QCD-based calculations to identify the ground state as the color-superconducting state [6-8].

We have learned the properties of high-temperature QCD matter from relativistic heavy-ion collision experiments, and such activities are going to be continued toward new regimes with larger baryon density in the Beam Energy Scan program. Besides, more and more experimental data are expected from the neutron star observation, which will be a promising probe into dense QCD matter. Interestingly, in both the heavy-ion collisions and the neutron star cores, dense matter is exposed to strong magnetic fields. As reviewed in Ref. [9], many interesting and exotic phenomena, mostly topological currents, are anticipated from the quantum anomaly as result of the coupling between the density and the magnetic field (see Ref. [10] for the implication to the heavy-ion phenomenology). In the context of neutron star physics, in which the magnetic field strength is of the order $10^{13}-10^{15} \mathrm{G}$ on the surface [11] and stronger inside, magnetic effects on color-superconducting quark matter have been studied [12-18]. Recently, in addition to possible topological currents, an important

\footnotetext{
fuku@nt.phys.s.u-tokyo.ac.jp

imaki@nt.phys.s.u-tokyo.ac.jp
}

Published by the American Physical Society under the terms of the Creative Commons Attribution 4.0 International license. Further distribution of this work must maintain attribution to the author(s) and the published article's title, journal citation, and DOI. Funded by SCOAP ${ }^{3}$. aspect of the chiral anomaly has been pointed out in Ref. [19]; the ground state structure as the chiral soliton lattice can be beautifully formulated for quark matter if the magnetic field is sufficiently strong (see Ref. [20] for a related discussion of the interplay between inhomogeneous quark matter and topological currents).

Various QCD phases are characterized by various condensates leading to the spontaneous breaking of global symmetries. Then, according to the broken symmetries, the physical vacuum has degeneracy, and the Nambu-Goldstone bosons are the dominant degrees of freedom for low-energy states. For manifold which can wrap nontrivially over configuration space, we can expect topologically stable solitons [21]. Owing to the rich variety of QCD phases, many different types of topological solitons may be present in nuclear and quark matter [22]. In this work we will specifically consider the domain-wall and the vortex or vorton.

On the one hand, the domain-wall is a two-dimensional object that is an interface between different energy minima. Some theoretical considerations hypothesize the presence of $\pi^{0}$ domain-walls in high-density nuclear matter. (It is also possible to think of three-dimensional pionic profiles, which are exemplified in a scenario of the Skyrme lattice [23].) Similarly, the $\eta$ domain-wall can stably exist in the color-flavor locked (CFL) color-superconducting phase $[24,25]$. It is important to note that a layered structure of the domain-walls is triggered by the anomaly-induced baryon charge on these axial domain-walls. Thus, keeping the total baryon charge, such a special configuration can reduce the system energy. We also mention another aspect of the anomaly; an anomaly-induced axial current at finite density [26] and magnetic moment on axial domain-walls [27] may cause spontaneous magnetization, which may be a microscopic origin of the strong magnetic field on magnetars (see also Refs. [28,29]).

We note that, as argued in Ref. [19], the periodically layered structure of the axial domain-walls is a QCD counterpart of "chiral soliton lattice" [30-33], which refers 
to special spin-aligned systems with breaking of parity and translational symmetry. The mathematical connection lies in the similarity between the Dzyaloshinskii-Moriya interaction in chiral materials [34] and the Wess-Zumino-Witten (WZW) action in QCD. This in turn means that the anomaly-induced phenomena suggested in the QCD context may be testable in analogous chiral materials.

The vortex, on the other hand, is a one-dimensional topological defect with a winding $\mathrm{U}(1)$ phase. One example is the nuclear vortex in superfluid nuclear matter, which could be continuously connected to the CFL vortex associated with $\mathrm{U}(1)_{\mathrm{B}}$ breaking [35]. Another example is the $\eta$ vortex in the CFL phase, which spontaneously breaks $\mathrm{U}(1)_{\mathrm{A}}$ by diquark condensate. Since these vortices carry angular momenta [36], rotating CFL matter, as may be realized in the neutron star cores [37], would form a lattice of these vortices. Like the anomaly-induced baryon charge on the domain-walls, we can also anticipate anomaly-induced currents on the vortices, such as an electric current along the $\eta$ vortex or an axial current along the baryon superfluid vortex [26,27]. Remarkably, such topological currents are nondissipative and persistent, which is unusual in the sense that the Bloch theorem, which forbids any persistent current in the ground state, is evaded [38].

In this work we will consider a vortex ring. Such a closed loop of vortex string is often referred to as a vorton. This special configuration could emerge at the edge of the domain-wall disk or just on its own. Here, we make a historical remark about the idea of the superconducting string [39] that invoked serious investigations on the cosmic string and possible vortons of the cosmic string during the phase transitions in the early Universe [40]. Now we would emphasize that the "QCD vorton" could exist as a stable object, especially in the CFL phase with kaon condensation [41]. There is a nonzero charge and a persistent superconducting current confined in the vortex core [42]. Because this persistent current is accompanied by finite angular momentum, the vorton is stabilized by the angular momentum conservation [43]. Moreover, a crucial difference of the CFL $K^{0}$ vorton from the cosmic string is that the vorton forms a domain-wall due to the small mass of the Nambu-Goldstone boson [44]. This complements a picture of the domain-wall disk surrounded by the vortex string, and its shape is sometimes described as the "drum vorton" in the literature $[41,45]$.

To investigate anomalous effects on QCD vortices, we will utilize the chiral effective theory. Because the low-energy dynamics is uniquely determined by the symmetry-breaking pattern, the formulation by the chiral effective theory is quite robust and does not require any microscopic details of nuclear or quark matter. The indispensable ingredient in theory is the WZW action to reproduce the quantum anomaly with low-energy degrees of freedom [46,47]. The best-known example of the WZW action is the description of the $\pi^{0} \rightarrow \gamma \gamma$ decay process [48] (see Ref. [49] for this process at high density). The full construction of the WZW action is discussed in Ref. [50], and it has been applied to the chiral magnetic effect in the hadronic phase [51].

We will find in this work that, in the presence of vortex singularities, the gauged WZW action requires new terms involving derivative commutators; otherwise, gauge invariance is violated and the charge conservation law is apparently broken. These terms turn out to incorporate anomalous currents absorbed or emitted by the vortex, which is microscopically carried by zeromodes sitting on the vortex.

As a concrete physical setup, we will examine two different kinds of finite-size axial domain-walls and vortices, namely, the $\pi^{0}$ vortex and the $\eta$ vortex, coupled with external magnetic fields. We will also argue that, with increasing magnetic fields, axial vortices absorb or emit the baryon and the electric charges onto the domain-wall. Interestingly, such a balance between the anomaly effect on the domain-wall in bulk and that on the vortex at edge has a clear interpretation as Callan-Harvey's mechanism of the anomaly inflow [52] once the axion vortex string is replaced with the $\pi^{0}$ vortex or the $\eta$ vortex.

This paper is organized as follows: In Sec. II, we will derive the chiral effective theory with the WZW action in the presence of the vortices. In Sec. III, we will investigate two concrete systems with the axial vortices and apply the fully gauge-invariant WZW action to see how the charge conservation is satisfied. In Sec. IV, we address the microscopic origin of the vortex currents in terms of quark degrees of freedom along the vortices. The final section $\mathrm{V}$ is devoted to the conclusion.

\section{TOPOLOGICAL CURRENT AND CONSERVATION LAW}

We introduce the chiral effective theory and the WZW action. In Sec. II A we extend the WZW action to a fully gauge-invariant form including vortex singularities. In Sec. II B we explicitly derive an extra contribution to the topological current from the gauge-invariant WZW action and discuss how the conservation law is satisfied on axial vortices.

\section{A. Chiral effective theory and currents}

The two-flavor chiral effective theory is characterized by the chiral Lagrangian given in terms of triplet $\Sigma$ of pseudoscalar Nambu-Goldstone bosons or pions $\pi_{i}$ 's. The nonanomalous part of the leading-order action is $S_{\chi}=\int d^{4} x \mathcal{L}_{\chi}$, where

$$
\mathcal{L}_{\chi}=\frac{f_{\pi}^{2}}{4} \operatorname{tr}\left(D_{\mu} \Sigma^{\dagger} D^{\mu} \Sigma+M \Sigma^{\dagger}+\Sigma M\right),
$$


with $D_{\mu} \Sigma=\partial_{\mu} \Sigma+i e A_{\mu}[Q, \Sigma]$ and $Q=\operatorname{diag}\left(\frac{2}{3},-\frac{1}{3}\right)$ in flavor space of $u$ - and $d$-quarks [53]. The differentiation of this action with respect to the electromagnetic field $A_{\mu}$ yields an electric current,

$j_{\mathrm{em}, \chi}^{\mu}=-\frac{\delta S_{\chi}}{\delta e A_{\mu}}=\frac{-i f_{\pi}^{2}}{2} \operatorname{tr}\left\{\frac{\tau^{3}}{2}\left[\Sigma D^{\mu} \Sigma^{\dagger}-\left(D^{\mu} \Sigma^{\dagger}\right) \Sigma\right]\right\}$.

In the above expression we chose a convention to take the derivative with $-e A_{\mu}$ for notational brevity. In physics language, $j_{\mathrm{em}, \chi}^{\mu}$ represents the electric current associated with $\pi^{ \pm}$flows. Here, we introduce several notations which we will frequently use in later discussions. Vector fields made with $\Sigma$ are commonly referred to as $L_{\mu}$ and $R_{\mu}$, defined by

$$
L_{\mu}=\Sigma \partial_{\mu} \Sigma^{\dagger}, \quad R_{\mu}=\left(\partial_{\mu} \Sigma^{\dagger}\right) \Sigma,
$$

with which we can further construct second-order tensors

$$
L_{\mu \nu}=\Sigma \partial_{\mu} \partial_{\nu} \Sigma^{\dagger}, \quad R_{\mu \nu}=\left(\partial_{\mu} \partial_{\nu} \Sigma^{\dagger}\right) \Sigma
$$

We note that $L_{\mu \nu}$ and $R_{\mu \nu}$ are symmetric tensors if the derivatives are commutative without vortex singularities.

Besides the nonanomalous current $j_{\mathrm{em}, \mathcal{\chi}}$, anomalous coupling between $\Sigma$ and external gauge fields leads to the topological current induced by quantum anomaly. This anomalous coupling is captured by the WZW action $[46,47]$. The five-dimensional compact form of the WZW action is well known as [47]

$$
S_{\mathrm{WZW}}^{(0)}=\frac{i}{80 \pi^{2}} \int_{\mathcal{D}} d^{5} x \epsilon^{\bar{\mu} \bar{\nu} \bar{\rho} \bar{\sigma}} \operatorname{tr}\left(L_{\bar{\mu}} L_{\bar{\nu}} L_{\bar{\rho}} L_{\bar{\sigma}} L_{\bar{\tau}}\right),
$$

in the absence of gauge fields. The indices with bar, $\bar{\mu}, \bar{\nu}, \bar{\rho}, \bar{\sigma}, \bar{\tau}$, run over five-dimensional spacetime denoted by $\mathcal{D}$. The fifth coordinate $x^{4} \in[0,1]$ is chosen such that $\partial \mathcal{D}=\mathcal{D}\left(x^{4}=1\right)=\mathcal{M}$, where $\mathcal{M}$ stands for physical fourdimensional spacetime.

Anomaly-induced electric current can be derived from the action (5), as discussed below. Under an infinitesimal $\mathrm{U}(1)_{\mathrm{em}}$ rotation, i.e., $\Sigma \rightarrow \Sigma+i \zeta[Q, \Sigma]$ and $A_{\mu} \rightarrow$ $A_{\mu}-\partial_{\mu} \zeta / e$, the action (5) varies up to the linear order in $\zeta$ as

$$
\delta S_{\mathrm{WZW}}^{(0)}=-\int_{\mathcal{M}} d^{4} x\left(\partial_{\mu} \zeta\right) X^{\mu}-\int_{\mathcal{D}} d^{5} x\left(\partial_{\bar{\mu}} \zeta\right) Y^{\bar{\mu}} .
$$

We note that the above decomposition of $X^{\mu}$ and $Y^{\bar{\mu}}$ is not unique. The most convenient choice for our discussions is as follows:

$$
X^{\mu}=-\frac{1}{48 \pi^{2}} \epsilon^{\mu \nu \rho \sigma} \operatorname{tr}\left(L_{\nu} L_{\rho} L_{\sigma}\right)
$$

$$
Y^{\bar{\mu}}=-\frac{1}{16 \pi^{2}} \epsilon^{\bar{\mu} \bar{\nu} \bar{\rho} \bar{\sigma} \bar{\tau}} \operatorname{tr}\left(L_{\bar{\nu}} L_{\bar{\rho}} L_{\bar{\sigma} \bar{\tau}}\right) .
$$

We made this choice such that $Y^{\bar{\mu}}$ would be vanishing for symmetric $L_{\bar{\sigma} \bar{\tau}}$.

We can modify the WZW action to make it gauge invariant up to the linear order as

$$
S_{\mathrm{WZW}}^{(1)}=S_{\mathrm{WZW}}^{(0)}-\int_{\mathcal{M}} d^{4} x e A_{\mu} X^{\mu}-\int_{\mathcal{D}} d^{4} x e A_{\bar{\mu}} Y^{\bar{\mu}}
$$

Yet this action is not fully gauge invariant beyond the leading order. We can reiterate the above procedures to the next order to obtain $S_{\mathrm{WZW}}^{(2)}$ with corrections to $X^{\mu}$ and $Y^{\bar{\mu}}$, which is in fact fully gauge invariant. Hereafter we will denote the fully gauge-invariant WZW action by $S_{\mathrm{WZW}}$.

In contrast, the baryon current cannot be obtained in the same way, since $\Sigma$ is not sensitive to $\mathrm{U}(1)_{\mathrm{B}}$ rotation. Instead, we identify the baryon current via the Gell-MannNishijima formula, $Q=I_{3}+B / 2$ [54], where $I_{3}$ and $B$ represent the isospin and the baryon number, respectively. The isospin part corresponds to nonanomalous $j_{\mathrm{em}, \chi}$ in Eq. (2), whereas $B$ arises from anomaly within the framework of the chiral effective theory. Accordingly, we should equate $B / 2$ and the anomalous part in $Q$. Further imposing the baryon conservation law [55], we can construct the gauged WZW action as

$$
\begin{aligned}
S_{\mathrm{WZW}}= & S_{\mathrm{WZW}}^{(0)}-\int_{\mathcal{M}} d^{4} x\left(\alpha_{\mu}+\frac{e}{2} A_{\mu}\right) j_{\mathrm{B}, \text { bulk }}^{\mu} \\
& -\int_{\mathcal{D}} d^{5} x\left(\alpha_{\bar{\mu}}+\frac{e}{2} A_{\bar{\mu}}\right) \mathrm{j}_{\mathrm{B}}^{\bar{\mu}} .
\end{aligned}
$$

Here a spurious gauge field $\alpha_{\mu}=\left(\mu_{\mathrm{B}}, \overrightarrow{0}\right)$ implements the coupling of the baryon chemical potential [56-58] with the topological currents given by

$$
\begin{aligned}
j_{\mathrm{B}, \mathrm{bulk}}^{\mu}= & -\frac{1}{24 \pi^{2}} \epsilon^{\mu \nu \rho \sigma} \\
& \times\left\{\operatorname{tr}\left(L_{\nu} L_{\rho} L_{\sigma}\right)-3 i e\left(\partial_{\nu} A_{\rho}\right) \operatorname{tr}\left[Q\left(L_{\sigma}+R_{\sigma}\right)\right]\right. \\
& \left.-3 i e A_{\nu} \operatorname{tr}\left[Q\left(L_{\rho} L_{\sigma}-R_{\rho} R_{\sigma}\right)\right]\right\}
\end{aligned}
$$

in the four-dimensional part of the action and

$$
\begin{aligned}
\mathrm{j}_{\mathrm{B}}^{\bar{\mu}}= & -\frac{1}{8 \pi^{2}} \epsilon^{\bar{\mu} \bar{\nu} \bar{\rho} \bar{\sigma} \bar{\tau}} \\
& \times\left\{\operatorname{tr}\left(L_{\bar{\nu}} L_{\bar{\rho}} L_{\bar{\sigma} \bar{\tau}}\right)-i e\left(\partial_{\bar{\nu}} A_{\bar{\rho}}\right) \operatorname{tr}\left[Q\left(L_{\bar{\sigma} \bar{\tau}}+R_{\bar{\sigma} \bar{\tau}}\right)\right]\right. \\
& \left.-i e A_{\bar{\nu}} \operatorname{tr}\left[Q\left(L_{\bar{\rho}} L_{\bar{\sigma} \bar{\tau}}-L_{\bar{\sigma} \bar{\tau}} L_{\bar{\rho}}-R_{\bar{\rho}} R_{\bar{\sigma} \bar{\tau}}+R_{\bar{\sigma} \bar{\tau}} R_{\bar{\rho}}\right)\right]\right\}
\end{aligned}
$$

in the five-dimensional part. We denote the ordinary fourdimensional current as $j_{\mathrm{B} \text {,bulk }}$, for it represents the current 
flowing not on vortices but in bulk. Here the first terms in Eqs. (11) and (12) come from $X^{\mu}$ in Eq. (7) and $Y^{\bar{\mu}}$ in Eq. (8) in the leading order. The four-dimensional current $j_{\mathrm{B}, \text { bulk }}^{\mu}$ is well known, which traces back to Ref. [46], whereas the five-dimensional $\mathrm{j}_{\mathrm{B}}^{\bar{\mu}}$ is a novel contribution in this work. One can immediately see that the fivedimensional current $\mathrm{j}_{\mathrm{B}}^{\bar{\mu}}$ would vanish unless $\Sigma$ has a singularity, i.e., $\left[\partial_{\bar{\mu}}, \partial_{\bar{\nu}}\right] \Sigma^{\dagger} \neq 0[59]$.

Finally, let us define the total baryon charge out of these currents. The baryon charge inferred from the ordinary four-dimensional current is

$$
Q_{\mathrm{B}, \mathrm{bulk}}=\int d^{3} \vec{x} j_{\mathrm{B}, \mathrm{bulk}}^{0},
$$

and another charge contribution from the five-dimensional current is

$$
Q_{\mathrm{B}, \mathrm{zm}}=\int d^{3} \vec{x} \int_{0}^{1} d x_{4} \mathrm{i}_{\mathrm{B}}^{0}
$$

As we will discuss later, $Q_{\mathrm{B}, \mathrm{zm}}$ appears from the zeromode contribution along vortices. By construction, the anomalyinduced electric charge is given by half of the baryon charge. In this paper, therefore, we will focus on baryon conservation only, from which the electric charge conservation naturally follows.

\section{B. Charge conservation}

If there is no singularity in $\Sigma$, the current conservation is satisfied in a simple way. That is, we see $\mathrm{j}_{\mathrm{B}}^{\bar{\mu}}=0$ in a reason mentioned right below Eq. (12) and

$$
\begin{aligned}
\partial_{\mu} j_{\mathrm{B}, \text { bulk }}^{\mu}= & -\frac{1}{8 \pi^{2}} \epsilon^{\mu \nu \rho \sigma} \\
& \times\left\{\operatorname{tr}\left(L_{\mu} L_{\nu} L_{\rho \sigma}\right)-i e\left(\partial_{\mu} A_{\nu}\right) \operatorname{tr}\left[Q\left(L_{\rho \sigma}+R_{\rho \sigma}\right)\right]\right. \\
& \left.-i e A_{\mu} \operatorname{tr}\left[Q\left(L_{\nu} L_{\rho \sigma}-L_{\rho \sigma} L_{\nu}-R_{\nu} R_{\rho \sigma}+R_{\rho \sigma} R_{\nu}\right)\right]\right\},
\end{aligned}
$$

which vanishes identically. Therefore, $Q_{\text {B.bulk }}$ by itself is conserved.

The situation would be, however, far more complicated when $\Sigma$ has a singularity leading to $\left[\partial_{\mu}, \partial_{\nu}\right] \Sigma^{\dagger} \neq 0$. Such a singularity is typically associated with the topological winding of vortex configurations. In the presence of a vortex flux, $\left[\partial_{\mu}, \partial_{\nu}\right] \Sigma^{\dagger}$ is nonzero proportional to the Dirac delta function at the vortex position. Then, we must conclude,

$$
\partial_{\mu} j_{\mathrm{B}, \text { bulk }}^{\mu} \neq 0
$$

and $Q_{\mathrm{B}, \text { bulk }}$ alone is no longer a conserved charge.
Let us see how this naive violation of the charge conservation law is cured by the five-dimensional contribution. By construction, the WZW action in Eq. (10) possesses local gauge symmetry in $\mathcal{D}$. Then, we can adapt gauge rotations in $\mathcal{D}$ in order not to affect $\mathcal{M}$, which leads to

$$
\partial_{\bar{\mu}} \mathrm{j}_{\mathrm{B}}^{\bar{\mu}}\left(x, x^{4}\right)=0 .
$$

Conversely, if we apply gauge rotations on $\partial \mathcal{D}=\mathcal{M}$ at $x^{4}=1$, we find

$$
\partial_{\mu} j_{\mathrm{B}, \text { bulk }}^{\mu}(x)=\mathfrak{j}_{\mathrm{B}}^{4}\left(x, x^{4}=1\right) .
$$

We can give a plain interpretation for Eq. (18) that the current $\dot{\mathrm{j}}_{\mathrm{B}}^{4}$ injected from $\mathcal{D}$ provides a source of the baryon charge in $\mathcal{M}$. We can rewrite Eq. (18) in a form of the current conservation by introducing the zeromode current, $j_{\mathrm{B}, \mathrm{zm}}^{\mu}$, as

$$
j_{\mathrm{B}, \mathrm{zm}}^{\mu}(x)=\int_{0}^{1} d x^{4} \mathrm{j}_{\mathrm{B}}^{\mu}\left(x, x^{4}\right)
$$

We will discuss the meaning of the "zeromode" later in Sec. IV. Then, using Eq. (17), we can rewrite the source term as

$$
\mathrm{i}_{\mathrm{B}}^{4}\left(x^{4}=1\right)=\int_{0}^{1} d x^{4} \partial_{4} \mathrm{j}_{\mathrm{B}}^{4}=-\partial_{\mu} j_{\mathrm{B}, \mathrm{Zm}}^{\mu}
$$

We note that $\dot{j}_{\mathrm{B}}^{4}\left(x^{4}=0\right)=0$ follows from Eq. (17). Finally we arrive at the baryon charge conservation law in the following concise form:

$$
\partial_{\mu}\left(j_{\mathrm{B}, \mathrm{bulk}}^{\mu}+j_{\mathrm{B}, \mathrm{zm}}^{\mu}\right)=0 .
$$

In fact, $Q_{\mathrm{B}, \mathrm{zm}}$ in Eq. (14) is nothing but the charge associated with $j_{\mathrm{B}, \mathrm{zm}}^{\mu}$, and obviously the total baryon charge,

$$
Q_{\mathrm{B}}=Q_{\mathrm{B}, \mathrm{bulk}}+Q_{\mathrm{B}, \mathrm{zm}},
$$

is the conserved quantity regardless of the presence of vortex singularities. As we will demonstrate in the next section, $j_{\mathrm{B}, \mathrm{zm}}^{\mu}$ corresponds to the baryon current carried by the vortex zeromode. Then, the nonconservation problem of $j_{\mathrm{B}, \text { bulk }}^{\mu}$ in bulk is resolved with $j_{\mathrm{B}, \mathrm{zm}}^{\mu}$ localized on vortex cores.

Let us make one remark on $j_{\mathrm{B}, \mathrm{zm}}^{\mu}$. The zeromode current (19) is unique only up to divergenceless terms. In fact, Eq. (19) implies that $j_{\mathrm{B}, \mathrm{zm}}^{\mu}$ may depend on the extension from $\mathcal{M}$ to $\mathcal{D}$, but it is clear from Eq. (20) that any difference caused by this would be irrelevant once the divergence is taken. 


\section{QCD AXIAL DOMAIN-WALLS AND VORTICES WITH MAGNETIC FIELDS}

So far, we have seen the general theory of conservation law in systems involving vortex singularities. In this section we address two concrete examples of such vortex configurations in QCD. One is the $\pi^{0}$ domain-wall and surrounding vortex as discussed in Sec. III A, and the other is the $\eta$ domain-wall and surrounding vortex as discussed in Sec. III B. In both cases a finite baryon number is anomalously induced with coupling to external magnetic fields.

\section{A. $\pi^{0}$ domain-wall and vortex}

As argued in Ref. [19], in extreme environments with sufficiently strong magnetic fields such as the neutron star cores, nuclear matter would form $\pi^{0}$ domain-wall layers. Once the $\pi^{0}$ domain-wall layers develop inside the neutron star, the edge of the $\pi^{0}$ domain-wall is a $\pi^{0}$ vortex string as illustrated in Fig. 1.

In the $\mathrm{QCD}$ vacuum the chiral condensate spontaneously breaks chiral symmetry as $\mathrm{SU}(2)_{\mathrm{L}} \times \mathrm{SU}(2)_{\mathrm{R}} \rightarrow \mathrm{SU}(2)_{\mathrm{V}}$ in the massless two-flavor case. External magnetic fields would explicitly break a part of chiral symmetry as $\mathrm{SU}(2)_{\mathrm{L}} \times \mathrm{SU}(2)_{\mathrm{R}} \rightarrow \mathrm{U}(1)_{\mathrm{A}} \times \mathrm{U}(1)_{\mathrm{V}}$, where this $\mathrm{U}(1)_{\mathrm{A}}$ symmetry has nothing to do with $\eta$ meson in the anomalous sector (which will be considered in Sec. III B) but corresponds to $\pi^{0}$. Therefore, in strongly magnetized quark matter, nonanomalous $\mathrm{U}(1)_{\mathrm{A}}$ symmetry is spontaneously broken by the chiral condensate. In this sense quark matter with strong magnetic fields could be regarded as a "chiral superfluid," which accommodates axial vortices as topological defects. If we consider a small but nonzero quark mass and place a ring of $\pi^{0}$ vortex in the system as in Fig. 1, the minimal surface area surrounded by the $\pi^{0}$ vortex string should form a $\pi^{0}$ domain-wall to minimize the energy cost by topological winding. Let us calculate $j_{\mathrm{B}, \mathrm{bulk}}^{\mu}$ and $j_{\mathrm{B}, \mathrm{zm}}^{\mu}$ in this setup.

First, we flash theoretical descriptions following Ref. [25]. It is convenient to use the following parametrization with $\chi, \theta, \phi$ :

$$
\Sigma=\cos \chi e^{i \tau_{3} \theta}+i \tau_{1} \sin \chi e^{i \tau_{3} \phi},
$$

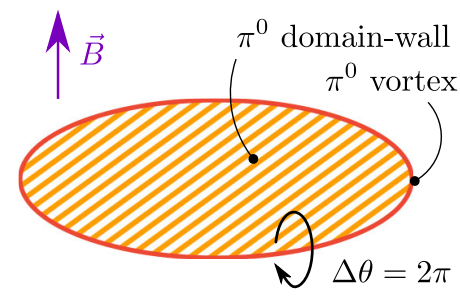

FIG. 1. $\pi^{0}$ domain-wall and surrounding $\pi^{0}$ vortex string at the edge. With increasing magnetic field piercing the domain-wall, more baryons are attached on the domain-wall according to Eq. (28). instead of conventional $\pi^{0}, \pi^{ \pm}$, where $\tau_{i}$ 's are Pauli matrices in two-flavor space. With this parametrization the nonanomalous Lagrangian (1) takes the form of

$$
\begin{aligned}
\mathcal{L}_{\chi}= & \frac{f_{\pi}^{2}}{2}\left[\left(\partial_{\mu} \chi\right)^{2}+\cos ^{2} \chi\left(\partial_{\mu} \theta\right)^{2}+\sin ^{2} \chi\left(\partial_{\mu} \phi-e A_{\mu}\right)^{2}\right. \\
& \left.-2 m_{\pi}^{2}(1-\cos \chi \cos \theta)\right],
\end{aligned}
$$

where $m_{\pi}$ represents the $\pi^{0}$ mass. We can easily see that this Lagrangian allows for a $\pi^{0}$ domain-wall. If we anticipate $\cos \chi \simeq 1$ holds away from the edge of the domain-wall for some reason, the Lagrangian (24) is reduced to the sineGordon model. The one-dimensional classical solution in the sine-Gordon model is well known to be

$$
\theta(z)=4 \arctan \left(e^{m_{\pi} z}\right)
$$

which has a smooth jump of $\theta$ by $2 \pi$ around $z \sim 0$. Hence, we shall call this special solution the $\pi^{0}$ domain-wall.

As we already pointed out in the beginning of this section, the $\pi^{0}$ domain-wall and vortex are topologically stabilized when strong magnetic fields are imposed [25]. In physics language, the strong magnetic field makes $\pi^{ \pm}$as massive as the imposed magnetic scale, which means $\cos \chi=1$ and $\sin \chi=0$ are energetically favored. Thus, for strongly magnetized quark matter, the sine-Gordon model is the genuine effective theory.

This $\pi^{0}$ domain-wall accompanies a $\pi^{0}$ vortex string along the edge. Since $\theta$ jumps by $2 \pi$ at the domain-wall, $\theta$ increments by $2 \pi$ along any small loop around the vortex (see Fig. 1). This winding on the vortex gives rise to a singularity, i.e., $\left[\partial_{i}, \partial_{j}\right] \theta=2 \pi \delta^{(2)}\left(x_{\perp}\right)$, where $x_{\perp}$ represents a transverse coordinate perpendicular to and centered at the vortex. It should be noted that $\sin ^{2} \chi=1$ on the vortex to avoid singularity behavior of the $\theta$ kinetic term. This implies that $\pi^{ \pm}$must be present around the $\pi^{0}$ vortex.

Next, let us evaluate the anomaly-induced baryon charge from the WZW action. The currents $j_{\mathrm{B} \text {,bulk }}^{\mu}$ in Eq. (11) and $j_{\mathrm{B}, \mathrm{zm}}^{\mu}$ in Eq. (19) are associated with the $\pi^{0}$ domain-wall and the $\pi^{0}$ vortex, respectively. We can readily compute $j_{\mathrm{B} \text {,bulk }}^{\mu}$ under the minimization condition of the kinetic energy, which concludes that $\partial_{\mu} \phi=e A_{\mu}$. Then only the second term in Eq. (11) remains finite, yielding

$$
j_{\mathrm{B}, \mathrm{bulk}}^{\mu}=\frac{e}{8 \pi^{2}} \epsilon^{\mu \nu \rho \sigma} F_{\nu \rho} \partial_{\sigma} \theta .
$$

The baryon density topologically induced by the anomaly is thus expressed as

$$
j_{\mathrm{B}, \text { bulk }}^{0}=\frac{e}{4 \pi^{2}} \vec{B} \cdot \vec{\partial} \theta .
$$

Alternatively, we can write down the integrated baryon number, $Q_{\text {B,bulk }}$ of Eq. (13), without even using the 
energy- minimization condition. In this case only the first term in Eq. (11) contributes to the spatial integration. Ultimately we find [60]

$$
Q_{\mathrm{B}, \mathrm{bulk}}=\frac{1}{2 \pi} \oint_{\mathrm{vortex}} d \phi .
$$

We can confirm the consistency between Eqs. (27) and (28) using $\partial_{\mu} \phi=e A_{\mu}$ and $\Delta \theta=2 \pi$. From this argument we understand that the integer baryon number populates on the $\pi^{0}$ domain-wall for quanta $2 \pi / e$ of the piercing magnetic flux.

We can also evaluate $j_{\mathrm{B}, \mathrm{zm}}^{\mu}$ in Eq. (19), and the condition $\partial_{\mu} \phi=e A_{\mu}$ makes the final result as simple as

$$
j_{\mathrm{B}, \mathrm{zm}}^{\mu}=-\frac{1}{4 \pi} \epsilon^{\mu \nu \rho \sigma} \partial_{\nu} \phi \delta_{\rho \sigma}^{(2)}\left(x_{\perp}\right) .
$$

Here, $\delta_{\rho \sigma}^{(2)}\left(x_{\perp}\right)$ represents the two-dimensional Dirac delta function on the $x_{\rho}-x_{\sigma}$ plane centered at the vortex position.

For example, if a vortex sits along the $z$-axis, $\delta_{x y}^{(2)}\left(x_{\perp}\right)=$ $-\delta_{y x}^{(2)}\left(x_{\perp}\right)=\delta(x) \delta(y)$ and $\delta_{x x}^{(2)}\left(x_{\perp}\right)=\delta_{y y}^{(2)}\left(x_{\perp}\right)=0$. Then the spatial volume integration of Eq. (29) amounts to

$$
Q_{\mathrm{B}, \mathrm{zm}}=\int d^{3} x j_{\mathrm{B}, \mathrm{zm}}^{0}=-\frac{1}{2 \pi} \oint_{\mathrm{vortex}} d \phi,
$$

which exactly cancels Eq. (28) to conform to the conservation law in the whole system. We have confirmed the balance between the bulk and the zeromode contributions to satisfy the conservation law. It is intriguing to argue that the balanced relation holds not only for the static case but also for more dynamical circumstances, as it should. To see this, let us imagine a physical setup with growing magnetic field with time. Such a time-dependent magnetic field should generate an eddy electric field via Faraday's law, and Eq. (26) leads to [61]

$$
\vec{j}_{\mathrm{B}, \text { bulk }}=-\frac{e}{8 \pi^{2}} \vec{E} \times \vec{\partial} \theta .
$$

Because $\vec{E}$ is along the polar angular direction and $\theta$ has spatial variation perpendicular to the $\pi^{0}$ domain-wall, the current is directed along the radial coordinate on the $\pi^{0}$ domain-wall, i.e., either inward to or outward from the $\pi^{0}$ vortex string where the baryon charge is absorbed or emitted.

\section{B. $\boldsymbol{\eta}$ domain-wall and vortex}

If anomalous $\mathrm{U}(1)_{\mathrm{A}}$ symmetry is effectively restored in extreme conditions to suppress instanton excitations, we can anticipate the spontaneous breaking of $\mathrm{U}(1)_{\mathrm{A}}$ and associated vortices with respect to $\eta$ mesons.

The most well-known example of extreme conditions to accommodate $\eta$ vortices is the color-flavor locked state of quark matter at high density [8]. Instantons are expected to be Debye screened by the density effect, and chiral symmetry, including $\mathrm{U}(1)_{\mathrm{A}}$, is spontaneously broken by the diquark condensates there. In this state the phase $\varphi_{\mathrm{A}}$ of $\Sigma=|\Sigma| e^{i \varphi_{\mathrm{A}}}$ is the $\eta_{0}$ meson (which we simply call $\eta$ in this paper) and becomes an Nambu-Goldstone boson. The effective Lagrangian for $\varphi_{\mathrm{A}}$ is given by $[24,62]$

$\mathcal{L}_{\eta}=\frac{f_{\eta}}{2}\left[\left(\partial_{0} \varphi_{\mathrm{A}}\right)^{2}-u^{2}\left(\vec{\partial} \varphi_{\mathrm{A}}\right)^{2}-2 m_{\eta}^{2}\left(1-\cos \varphi_{\mathrm{A}}\right)\right]$,

where $f_{\eta}$ and $m_{\eta}$ are the decay constant and the mass of the $\eta$ meson. We note that $u^{2}$ represents the speed of $\eta$ and is not necessarily the unity in a medium which breaks Lorentz symmetry.

The Lagrangian (32) is of the sine-Gordon type and admits an $\eta$ domain-wall solution characterized by a jump of $\varphi_{\mathrm{A}}$ by $2 \pi$. In the same way as the $\pi^{0}$ domain-wall, this $\eta$ domain-wall also accompanies the $\eta$ vortex string at the edge, leading to $\left[\partial_{i}, \partial_{j}\right] \varphi_{\mathrm{A}}=2 \pi \delta^{(2)}\left(x_{\perp}\right)$ at the vortex core.

The rest of the discussion goes parallel to the previous subsection. We can evaluate $j_{\mathrm{B}, \mathrm{bulk}}^{\mu}$ and $j_{\mathrm{B}, \mathrm{zm}}^{\mu}$ associated with the $\eta$ domain-wall and the $\eta$ vortex, respectively. Then, a straightforward computation results in the following expression [27]:

$$
j_{\mathrm{B}, \text { bulk }}^{\mu}=\frac{e}{24 \pi^{2}} \epsilon^{\mu \nu \rho \sigma} F_{\nu \rho} \partial_{\sigma} \varphi_{\mathrm{A}},
$$

which is only factor $1 / 3$ different from Eq. (26). Actually, from the microscopic point of view, the difference between $\pi^{0}$ and $\eta$ is only a relative sign between $\bar{u} u$ and $\bar{d} d$. We can explain this factor difference between $\frac{2}{3} e-\left(-\frac{1}{3} e\right)=e$ and $\frac{2}{3} e+\left(-\frac{1}{3} e\right)=\frac{1}{3} e$. We find the zeromode contribution as

$$
j_{\mathrm{B}, \mathrm{zm}}^{\mu}=-\frac{e}{12 \pi} \epsilon^{\mu \nu \rho \sigma} A_{\nu} \delta_{\rho \sigma}^{(2)}\left(x_{\perp}\right) .
$$

Using $\partial_{\mu} \phi=e A_{\mu}$, we see that Eq. (34) is only factor $1 / 3$ different from Eq. (29).

Before closing this subsection, let us point out an interesting observation which motivates our analysis in the next section. If we take the divergence of the zeromode current, we will have a relation analogous to the anomaly; i.e., we immediately get the following from Eq. (34):

$$
\partial_{\mu} j_{\mathrm{B}, \mathrm{zm}}^{\mu}=-\frac{e}{24 \pi} \epsilon^{\mu \nu \rho \sigma} F_{\mu \nu} \delta_{\rho \sigma}^{(2)}\left(x_{\perp}\right)
$$

In fact, along the vortex as dictated by $\delta_{\rho \sigma}^{(2)}\left(x_{\perp}\right)$, the above is nothing but the gauge anomaly relation for $(1+1)$ dimensional chiral fermions with a total charge of $\frac{2}{3} e-\frac{1}{3} e=\frac{1}{3} e$. Such formal similarity is not accidental but is attributed to physical contents of the vortex in terms of chiral $u$ - and $d$-quarks, as we discuss in the next section. 


\section{ANOMALY INFLOW}

It may appear nontrivial that our arguments in the previous section, based on the gauged WZW action, are related to the zeromode contributions from the fermionic sector. To fill in this gap, we shall make a brief review on Callan-Harvey's mechanism of the anomaly inflow on axion vortices following Ref. [52].

We introduce axion $a(x)$ coupled to fermions as

$$
S=\int d^{4} x \bar{\psi}\left[i \gamma^{\mu}\left(\partial_{\mu}+i e A_{\mu}\right)-G \Phi\right] \psi,
$$

with $\Phi=|\Phi| e^{i \gamma_{5} a}$ [63]. The coupling constant $G$ will turn out to be irrelevant in what follows. Here, we consider only one flavor for simplicity, but generalization is straightforward. Suppose that the symmetry with respect to the $a$ rotation (that is, Peccei-Quinn symmetry) is spontaneously broken to set $|\Phi|=1$. The current expectation value is expressed by the fermion propagator as

$$
j^{\mu}(x)=-\operatorname{tr}\left[\gamma^{\mu} P(x, y \rightarrow x)\right],
$$

where the concrete form of the propagator is $P=$ $i /\left(i \gamma^{\mu} \partial_{\mu}-G \Phi\right)$. In the long wavelength limit, $G \rightarrow \infty$, we can expand the propagator in terms of $1 / G$, and a nonzero contribution remains as

$$
\begin{aligned}
j^{\mu}(x) & =-2 e \operatorname{tr}\left[\gamma^{\mu} \gamma^{\nu} \gamma^{\rho} \gamma^{\sigma} \gamma_{5}\right] \partial_{\nu} A_{\rho} \partial_{\sigma} a \int \frac{d^{4} p}{(2 \pi)^{4}} \frac{G^{2}}{\left(p^{2}-G^{2}\right)^{3}} \\
& =\frac{e}{8 \pi^{2}} \epsilon^{\mu \nu \rho \sigma} F_{\nu \rho} \partial_{\sigma} a,
\end{aligned}
$$

whose divergence reads

$$
\partial_{\mu} j^{\mu}=\frac{e}{8 \pi^{2}} \epsilon^{\mu \nu \rho \sigma} F_{\nu \rho} \partial_{\mu} \partial_{\sigma} a .
$$

One might think that this divergence vanishes, but this is not the case when an "axion vortex string" is present, which causes the singularity $\left[\partial_{\mu}, \partial_{\sigma}\right] a=2 \pi \delta_{\mu \sigma}^{(2)}\left(x_{\perp}\right)$, yielding

$$
\partial_{\mu} j^{\mu}=\frac{e}{8 \pi} \epsilon^{\mu \nu \rho \sigma} F_{\mu \nu} \delta_{\rho \sigma}^{(2)}\left(x_{\perp}\right),
$$

which apparently indicates violation of the gauge symmetry.

The key ingredient to resolve this apparent puzzle comes from a zeromode in the fermionic sector, which emerges in the presence of the axion vortex [39,63]. If the Dirac operator, $\left(i \gamma^{\mu} \partial_{\mu}-G \Phi\right)$, has a zero eigenvalue, we cannot take the inversion in the evaluation of Eq. (37) and should separately calculate the zeromode contribution,

$$
j_{\mathrm{zm}}^{\mu}=\bar{\psi}_{\mathrm{zm}} \gamma^{\mu} \psi_{\mathrm{zm}} .
$$

Here, $\psi_{\mathrm{zm}}$ represents the zeromode solution of the Dirac equation. To help our intuitive understanding, let us concretely specify the vortex configuration. Below we make use of the cylindrical coordinates $(r, z, \varphi)$ and place an axial vortex, $a(\varphi)=+\varphi$, along the $z$-axis at $r=0$. Then the zeromode equations read

$$
\left[i \gamma^{\alpha} \partial_{\alpha}+i \gamma^{1}\left(\cos \varphi+\gamma^{1} \gamma^{2} \sin \varphi\right) \partial_{r}\right] \psi_{\mathrm{zm}, \mathrm{L}}=G|\Phi| e^{i a} \psi_{\mathrm{zm}, \mathrm{R}},
$$

$$
\left[i \gamma^{\alpha} \partial_{\alpha}+i \gamma^{1}\left(\cos \varphi+\gamma^{1} \gamma^{2} \sin \varphi\right) \partial_{r}\right] \psi_{\mathrm{zm}, \mathrm{R}}=G|\Phi| e^{-i a} \psi_{\mathrm{zm}, \mathrm{L}}
$$

where 1 and 2 of $\gamma^{\mu}$ refer to the transverse directions perpendicular to the $z$ or 3 direction. The index $\alpha$ runs over 0 and 3 only. We can solve these equations to get the zeromode solutions as

$$
\psi_{\mathrm{zm}, \mathrm{L}}=\exp \left[-\int_{0}^{r} d r^{\prime} G|\Phi|\left(r^{\prime}\right)\right] \xi(t, z)
$$

and $\psi_{\mathrm{zm}, \mathrm{R}}=-i \gamma^{1} \psi_{\mathrm{zm}, \mathrm{L}}$. Here, $\xi(t, z)$ is a solution of the free Dirac equation, i.e.,

$$
\gamma^{\alpha} \partial_{\alpha} \xi=0
$$

with right-handed "chirality" defined by $\gamma^{0} \gamma^{3} \xi=+\xi$, which implies that $\left(\partial_{0}+\partial_{z}\right) \xi=0$. The exponential factor in Eq. (43) shows that this solution is localized near the axion vortex string and approaches $\delta^{(2)}\left(x_{\perp}\right)$ in the limit $G \rightarrow \infty$. Then the $(1+1)$-dimensional effective action for the zeromode on the axial vortex takes the following form:

$$
S_{\mathrm{zm}}=\int d t d z \bar{\xi}\left[i \gamma^{\alpha}\left(\partial_{\alpha}+i e A_{\alpha}\right)\right] \xi
$$

where we retrieved the gauge field in the covariant derivative. This $(1+1)$-dimensional theory exhibits the gauge anomaly to conclude $\partial_{\alpha} j_{\text {zm }}^{\alpha}=-\left(e E_{z} / 2 \pi\right) \delta^{(2)}\left(x_{\perp}\right)$. It is a straightforward exercise to reexpress this result in a covariant manner, which finally yields

$$
\partial_{\mu} j_{\mathrm{zm}}^{\mu}=-\frac{e}{8 \pi} \epsilon^{\mu \nu \rho \sigma} F_{\mu \nu} \delta_{\rho \sigma}^{(2)}\left(x_{\perp}\right) .
$$

In this way the apparent violation of the gauge symmetry in Eq. (40) is precisely canceled by the zeromode contribution of Eq. (46). This means that if we take account of both the "inflow" of Eq. (40) in bulk and the "leakage" of Eq. (46) from the edge, the whole system conserves the charge as it should.

Let us then reinterpret the arguments above in the language of the effective action. As we shall see immediately, the effective action of the system involving axion vortices is given by the following five-dimensional form: 


$$
S_{\text {axion }}=-\frac{e^{2}}{8 \pi^{2}} \int_{\mathcal{D}} d^{5} x \epsilon^{\bar{\mu} \bar{\nu} \bar{\rho} \bar{\sigma} \bar{\tau}} F_{\bar{\mu} \bar{\nu}} F_{\bar{\rho} \bar{\sigma}} \partial_{\bar{\tau}} a
$$

To see this, decompose this action into the four- and five-dimensional pieces, a process that is reminiscent of Eq. (10). That is,

$$
S_{\text {axion }}=-\int_{\mathcal{M}} d^{4} x e A_{\mu} j^{\mu}-\int_{\mathcal{D}} d^{5} x e A_{\bar{\mu}} \overline{\mathrm{i}}^{\bar{\mu}}
$$

with the four-dimensional current $j^{\mu}$ given by Eq. (38) and the five-dimensional current $j^{\bar{\mu}}$ defined by

$$
\mathrm{j}^{\bar{\mu}}=\frac{e}{8 \pi^{2}} \epsilon^{\bar{\mu} \bar{\nu} \bar{\sigma} \bar{\tau}} F_{\bar{\nu} \bar{\rho}} \partial_{\bar{\sigma}} \partial_{\bar{\tau}} a .
$$

The gauge invariance of the whole action assures that the five-dimensional part in Eq. (48) cancels the gauge variance of the other term in the presence of vortex singularity. This five-dimensional term thus reproduces the gauge anomaly of the chiral zeromode given by Eq. (45) [64]. This cancellation ensures that the zeromode current, defined in the same manner as Eq. (19), gives a divergence identical to Eq. (46).

Now the analogy to the case with the QCD axial vortices is clear; the five-dimensional action in Eq. (10), derived from Eq. (5), gives the consistent effective action inclusive of the anomalous action of fermionic zeromodes. Indeed, for the systems we considered in Sec. III, we could explicitly check the consistency about $j_{\mathrm{B}, \mathrm{zm}}^{\mu}$ from the zeromode construction and that from the effective action approach. For the $\eta$ vortex, Eq. (35) is immediately reproduced from Eq. (46). Interested readers can further consult the Appendix for the microscopic derivation of the zeromode current.

\section{CONCLUSIONS}

We considered a general problem of gauge invariance of the WZW action for singular configurations which make the order of derivatives noncommutative. This is not an academic subject, but it provides us with insight to understand how the topologically induced charges should be conserved.

As concrete examples we discussed the $\pi^{0}$ domain-wall and vortex and the $\eta$ domain-wall and vortex. In the presence of an external magnetic field the baryon and the electric charges are attached to the domain-walls due to the anomaly coupling. We explicitly confirmed that such induced charges are precisely canceled by singular contributions from the domain-wall edge, that is, the surrounding vortex ring or the vorton.

Interestingly, this cancellation mechanism has the same theoretical structure as Callan-Harvey's mechanism of the anomaly inflow for the axion vortex; the anomaly on the domain-wall in bulk is canceled by another anomaly in the $(1+1)$-dimensional fermionic sector of the vortex.

In the present work we limited ourselves to the twoflavor case, and the three-flavor extension would be an intriguing future problem. Then, it is known that the genuine ground state of dense quark matter is definitely the CFL phase if the baryon density is large enough. Since the CFL phase is a superfluid, it accommodates topologically stable vortices. Thanks to color and flavor degrees of freedom, many different types of vortices may appear. Some changes in the linkage number of various vortex entanglements could cause new topological effects, in the same way as the chiral magnetic effect associated with the linkage number change in terms of the magnetic fluxes [65]. A possible realization of the vortex linkage is found in the CFL phase with kaon condensation which supports vortons, i.e., topologically stable vortex rings [41-43]. Mixed condensates of $K^{0}$ and $K^{+}$[66] lead to both $K^{0}$ and $K^{+}$vortices. Since a $K^{+}$vortex carries a magnetic flux, a nontrivial linkage between the $K^{0}$ and $K^{+}$vortices would correspond to a situation in which a magnetic flux pierces a vorton [42], as we considered in this work. For the microscopic description of the charge density and currents in such systems, the extra terms involving the derivative commutator that we found in this work would play an essential role. The anomaly inflow in the context of dense QCD with vortices would certainly merit further investigation.

\section{ACKNOWLEDGMENTS}

The authors thank Naoki Yamamoto and Ho-Ung Yee for useful discussions and conversations. This work was supported by Japan Society for the Promotion of Science (JSPS) KAKENHI Grants No. 15H03652 and No. 15K13479.

\section{APPENDIX: MICROSCOPIC DERIVATION OF THE ZEROMODE CURRENT FOR THE $\pi^{0}$ VORTEX}

We begin with the fermionic action for quarks,

$$
S=\int d^{4} x\left[i \bar{q} \gamma^{\mu} \partial_{\mu} q-G\left(\bar{q}_{\mathrm{L}} \Sigma q_{\mathrm{R}}+\bar{q}_{\mathrm{R}} \Sigma^{\dagger} q_{\mathrm{L}}\right)\right],
$$

where $q$ represents the quark field and $G$ is the Yukawa coupling constant, which we will send to infinity in the end of the calculation. In cylindrical coordinates $(r, z, \varphi)$, a $\pi^{0}$ vortex lying along the $z$-axis is parametrized by

$$
\Sigma=\cos \chi(r) e^{i \tau_{3} \theta(\varphi)}+i \tau_{1} \sin \chi(r) e^{i \tau_{3} \phi(t, z)},
$$

with $\theta(\varphi)=+\varphi$, and the boundary condition $\cos \chi \rightarrow 1$ for $r \rightarrow \infty$ should be imposed. The zeromode equations corresponding to Eqs. (42a) and (42b) are 


$$
\begin{gathered}
{\left[i \gamma^{\alpha} \partial_{\alpha}+i \gamma^{1}\left(\cos \varphi+\gamma^{1} \gamma^{2} \sin \varphi\right) \partial_{r}\right] q_{\mathrm{L}}} \\
=G\left(\cos \chi e^{i \tau_{3} \varphi}+i \sin \chi e^{i \tau_{3} \phi}\right) q_{\mathrm{R}}, \\
{\left[i \gamma^{\alpha} \partial_{\alpha}+i \gamma^{1}\left(\cos \varphi+\gamma^{1} \gamma^{2} \sin \varphi\right) \partial_{r}\right] q_{\mathrm{R}}} \\
=G\left(\cos \chi e^{i \tau_{3} \varphi}+i \sin \chi e^{i \tau_{3} \phi}\right) q_{\mathrm{L}},
\end{gathered}
$$

where $\alpha=0,3$. We can write down an explicit solution for sufficiently large $G$ as

$$
q_{\mathrm{L}}=\exp \left[-\int_{0}^{r} d r^{\prime} G \cos \chi\left(r^{\prime}\right)\right] \xi(t, z)
$$

and $q_{\mathrm{R}}=-i \gamma^{1} q_{\mathrm{L}}$. Here, $\xi(t, z)$ satisfies not the free Dirac equation but the equation with $\phi$ background; that is,

$$
\left(i \gamma^{\alpha} \partial_{\alpha}-G \tau_{1} \gamma^{1} e^{i \tau_{3} \phi}\right) \xi=0 .
$$

We note that the chirality should be flipped depending on the quark flavors, i.e., $\tau_{3} \gamma^{0} \gamma^{3} \xi=+\xi$. The exponential factor is reduced to $\delta^{(2)}\left(x_{\perp}\right)$ in the limit of $G \rightarrow \infty$. To sort expressions out, it would be convenient to introduce a new set of Dirac matrices,

$$
\Gamma^{0}=i \tau_{1} \gamma^{2} \gamma^{3}, \quad \Gamma^{3}=i \tau_{1} \gamma^{2} \gamma^{0}, \quad \Gamma_{5}=\tau_{3} .
$$

Under the constraint, $\tau_{3} \gamma^{0} \gamma^{3}=+1$, we can prove the Clifford algebra, $\left\{\Gamma^{\alpha}, \Gamma^{\beta}\right\}=2 g^{\alpha \beta}, \quad\left\{\Gamma^{\alpha}, \Gamma_{5}\right\}=0$, and
$\left(\Gamma_{5}\right)^{2}=1$. Then, the $(1+1)$-dimensional effective action for the zeromode on the $\pi^{0}$ vortex takes the form

$$
S_{\mathrm{zm}}=\int d t d z \bar{\xi}\left(i \Gamma^{\alpha} \partial_{\alpha}-G e^{i \Gamma_{5} \phi}\right) \xi
$$

with $\bar{\xi}=\xi^{\dagger} \Gamma^{0}$. The most efficient method to infer the current from this effective action is to rewrite this into the bosonized form in terms of boson field $\sigma$, namely $S_{\mathrm{zm}} \rightarrow S_{\mathrm{zm}}^{\prime}$, with

$$
S_{\mathrm{zm}}^{\prime}=N_{\mathrm{c}} \int d t d z\left[\frac{1}{8 \pi} \partial_{\mu} \sigma \partial^{\mu} \sigma-G \cos (\sigma-\phi)\right],
$$

where $N_{\mathrm{c}}$ is the color number of quarks [60]. The $(1+1)$ dimensional quark current is given by $j^{\alpha}=\left(N_{\mathrm{c}} / 2 \pi\right) \epsilon^{\alpha \beta} \partial_{\beta} \sigma$. From the minimum of the potential $G \cos (\sigma-\phi)$, we should plug $\sigma=\phi$ into this expression. The overall factor $N_{\mathrm{c}}$ is eliminated by the conversion from the quark current to the baryon current in which we are interested, and taking account of $\delta^{(2)}\left(x_{\perp}\right)$ for the current in the original $(3+1)$ dimensional theory, we finally arrive at

$$
j_{\mathrm{B}, \mathrm{zm}}^{\mu}=-\frac{1}{4 \pi} \epsilon^{\mu \nu \rho \sigma} \partial_{\nu} \phi \delta_{\rho \sigma}^{(2)}\left(x_{\perp}\right)
$$

which is nothing but Eq. (29).
[1] K. Rajagopal and F. Wilczek, in At the Frontier of Particle Physics, edited by M. Shifman (World Scientific, Singapore, 2001), Vol. 3, 2061.

[2] M. G. Alford, A. Schmitt, K. Rajagopal, and T. Schäfer, Rev. Mod. Phys. 80, 1455 (2008).

[3] K. Fukushima and T. Hatsuda, Rep. Prog. Phys. 74, 014001 (2011).

[4] K. Fukushima and C. Sasaki, Prog. Part. Nucl. Phys. 72, 99 (2013).

[5] M. Buballa and S. Carignano, Prog. Part. Nucl. Phys. 81, 39 (2015).

[6] R. Rapp, T. Schäfer, E. V. Shuryak, and M. Velkovsky, Phys. Rev. Lett. 81, 53 (1998).

[7] M. G. Alford, K. Rajagopal, and F. Wilczek, Phys. Lett. 422B, 247 (1998).

[8] M. Alford, C. Kouvaris, and K. Rajagopal, Phys. Rev. Lett. 92, 222001 (2004).

[9] V. A. Miransky and I. A. Shovkovy, Phys. Rep. 576, 1 (2015).

[10] D. E. Kharzeev, J. Liao, S. A. Voloshin, and G. Wang, Prog. Part. Nucl. Phys. 88, 1 (2016).

[11] A. K. Harding and D. Lai, Rep. Prog. Phys. 69, 2631 (2006).
[12] M. G. Alford, J. Berges, and K. Rajagopal, Nucl. Phys. B571, 269 (2000).

[13] E. J. Ferrer, V. de la Incera, and C. Manuel, Phys. Rev. Lett. 95, 152002 (2005).

[14] E. J. Ferrer, V. de la Incera, and C. Manuel, Nucl. Phys. B747, 88 (2006).

[15] E. J. Ferrer and V. de la Incera, Phys. Rev. Lett. 97, 122301 (2006).

[16] E. J. Ferrer and V. de la Incera, Phys. Rev. D 76, 045011 (2007).

[17] J. L. Noronha and I. A. Shovkovy, Phys. Rev. D 76, 105030 (2007); 86, 049901E (2012).

[18] K. Fukushima and H. J. Warringa, Phys. Rev. Lett. 100, 032007 (2008).

[19] T. Brauner and N. Yamamoto, J. High Energy Phys. 04 (2017) 132.

[20] K. Fukushima and P. Morales, Phys. Rev. Lett. 111, 051601 (2013).

[21] N. S. Manton and P. Sutcliffe, Topological Solitons (Cambridge University Press, Cambridge, 2007).

[22] M. Eto, Y. Hirono, M. Nitta, and S. Yasui, Prog. Theor. Exp. Phys. 2014, 012D01 (2014). 
[23] H. Forkel, A. D. Jackson, M. Rho, C. Weiss, A. Wirzba, and H. Bang, Nucl. Phys. A504, 818 (1989).

[24] D. T. Son, M. A. Stephanov, and A. R. Zhitnitsky, Phys. Rev. Lett. 86, 3955 (2001).

[25] D. T. Son and M. A. Stephanov, Phys. Rev. D 77, 014021 (2008).

[26] M. A. Metlitski and A. R. Zhitnitsky, Phys. Rev. D 72, 045011 (2005).

[27] D. T. Son and A. R. Zhitnitsky, Phys. Rev. D 70, 074018 (2004).

[28] E. Nakano and T. Tatsumi, Phys. Rev. D 71, 114006 (2005); T. Tatsumi, T. Maruyama, E. Nakano, and K. Nawa, Nucl. Phys. A774, 827 (2006).

[29] M. Eto, K. Hashimoto, and T. Hatsuda, Phys. Rev. D 88, 081701 (2013); K. Hashimoto, Phys. Rev. D 91, 085013 (2015).

[30] I. Dzyaloshinskii, Sov. Phys. JETP 19, 17 (1964).

[31] Y. Togawa, T. Koyama, K. Takayanagi, S. Mori, Y. Kousaka, J. Akimitsu, S. Nishihara, K. Inoue, A. S. Ovchinnikov, and J. Kishine, Phys. Rev. Lett. 108, 107202 (2012).

[32] J.-i. Kishine and A. Ovchinnikov, in Solid State Physics, edited by R. E. Camley and R. L. Stamps (Elsevier, Cambridge, 2015), Vol. 66, pp. 1-130.

[33] Y. Togawa, Y. Kousaka, S. Nishihara, K. Inoue, J. Akimitsu, A. Ovchinnikov, and J.-i. Kishine, Phys. Rev. Lett. 111, 197204 (2013).

[34] I. Dzyaloshinsky, J. Phys. Chem. Solids 4, 241 (1958); T. Moriya, Phys. Rev. 120, 91 (1960).

[35] M. M. Forbes and A. R. Zhitnitsky, Phys. Rev. D 65, 085009 (2002).

[36] R. L. Davis and E. P. S. Shellard, Phys. Rev. Lett. 63, 2021 (1989).

[37] M. G. Alford, J. A. Bowers, and K. Rajagopal, J. Phys. G: Nucl. Part. Phys. 27, 541 (2001).

[38] N. Yamamoto, Phys. Rev. D 92, 085011 (2015).

[39] E. Witten, Nucl. Phys. B249, 557 (1985).

[40] T. W. B. Kibble, Phys. Rep. 67, 183 (1980); R. L. Davis and E. P. S. Shellard, Nucl. Phys. B323, 209 (1989); J. Garaud, E. Radu, and M. S. Volkov, Phys. Rev. Lett. 111, 171602 (2013).

[41] K. B. W. Buckley and A. R. Zhitnitsky, J. High Energy Phys. 08 (2002) 013.
[42] D. B. Kaplan and S. Reddy, Phys. Rev. Lett. 88, 132302 (2002).

[43] K. B. W. Buckley, M. A. Metlitski, and A. R. Zhitnitsky, Phys. Rev. D 68, 105006 (2003).

[44] D. T. Son, arXiv:hep-ph/0108260.

[45] B. Carter, R. H. Brandenberger, and A.-C. Davis, Phys. Rev. D 65, 103520 (2002).

[46] J. Wess and B. Zumino, Phys. Lett. 37B, 95 (1971).

[47] E. Witten, Nucl. Phys. B223, 422 (1983).

[48] S. L. Adler, Phys. Rev. 177, 2426 (1969); J. S. Bell and R. Jackiw, Nuovo Cimento A 60, 47 (1969).

[49] M. A. Nowak, M. Rho, A. Wirzba, and I. Zahed, Phys. Lett. B 497, 85 (2001).

[50] R. Kaiser and H. Leutwyler, Eur. Phys. J. C 17, 623 (2000); R. Kaiser, Phys. Rev. D 63, 076010 (2001).

[51] K. Fukushima and K. Mameda, Phys. Rev. D 86, 071501 (2012).

[52] C. G. Callan, Jr. and J. A. Harvey, Nucl. Phys. B250, 427 (1985).

[53] J. Gasser and H. Leutwyler, Ann. Phys. (N.Y.) 158, 142 (1984).

[54] T. Nakano and K. Nishijima, Prog. Theor. Phys. 10, 581 (1953); M. Gell-Mann, Nuovo Cimento 4, 848 (1956).

[55] C. Vafa and E. Witten, Nucl. Phys. B234, 173 (1984).

[56] J. B. Kogut, M. A. Stephanov, and D. Toublan, Phys. Lett. B 464, 183 (1999).

[57] K. Splittorff, D. T. Son, and M. A. Stephanov, Phys. Rev. D 64, 016003 (2001).

[58] D. T. Son, arXiv:hep-ph/0204199.

[59] T. Kalaydzhyan, Phys. Rev. D 89, 105012 (2014).

[60] J. Goldstone and F. Wilczek, Phys. Rev. Lett. 47, 986 (1981).

[61] N. Yamamoto, Phys. Rev. D 93, 085036 (2016).

[62] T. Schäfer, Phys. Rev. D 65, 094033 (2002).

[63] R. D. Peccei and H. R. Quinn, Phys. Rev. Lett. 38, 1440 (1977); R. Jackiw and P. Rossi, Nucl. Phys. B190, 681 (1981).

[64] M. Stone, Ann. Phys. (N.Y.) 207, 38 (1991).

[65] Y. Hirono, D. E. Kharzeev, and Y. Yin, Phys. Rev. Lett. 117, 172301 (2016).

[66] D. B. Kaplan and S. Reddy, Phys. Rev. D 65, 054042 (2002). 